\title{
Noncommutative Integrability and Recursion Operators 4
}

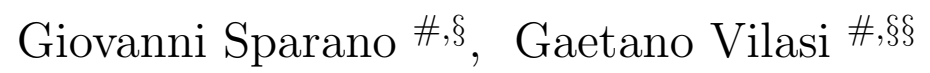 \\ \# Erwin Schrœdinger International Institute for Mathematical Physics, \\ Boltzmanngasse 4, A-1090 Wien, Austria. \\ \# Istituto Nazionale di Fisica Nucleare, Gruppo Collegato di Salerno, Italy. \\ $\S$ Dipartimento di Matematica e Informatica, Salerno University, Italy. \\ $\S \S$ Dipartimento di Scienze Fisiche "E.R.Caianiello",Salerno University, \\ Italy
}

\begin{abstract}
Geometric structures underlying commutative and non commutative integrable dynamics are analyzed. They lead to a new characterization of noncommutative integrability in terms of spectral properties and of Nijenhuis torsion of an invariant $(1,1)$ tensor field. The construction of compatible symplectic structures is also discussed.

Subj. Class.: Dynamical Systems.

1991 MSC: 58F05

Keywords: Noncommutative integrability; Recursion Operators; biHamiltonian Dynamics.

\footnotetext{
${ }^{1}$ Supported in part by the italian Ministero dell' Università e della Ricerca Scientifica e Tecnologica.

Corresponding author: G. Vilasi, Dipartimento di Scienze Fisiche "E. R. Caianiello", via S. Allende, 84081-Baronissi (Salerno), Italy.

Phone: 039-089-965317; Fax: 039-089-965275; e-mail: vilasi@sa.infn.it
} 


\section{Introduction}

In the last few decades there has been a renewed interest in completely integrable Hamiltonian systems, whose concept goes back to the last century [24] and which, loosely speaking, are dynamical systems admitting a Hamiltonian description and possessing sufficiently many constants of motion, so that they can be integrated by quadratures. Some qualitative features of these systems remain true in some special classes of infinite-dimensional Hamiltonian systems expressed by nonlinear evolution equations as, for instance, Korteweg-de Vries and sine-Gordon [33].

A relevant progress in the study of these systems with an infinite-dimensional phase manifold $\mathcal{M}$, was the introduction of the Lax Representation [22] which played an important role in formulating the Inverse Scattering Method, universally recognized as one of the most remarkable result of theoretical physics in last decades, and of the AKNS scheme [1]. This method allows the integration of non linear dynamics, both with a finitely or infinitely many degrees of freedom, for which a Lax representation can be given [16], this being both of physical and mathematical relevance [31].

Most of the evolution equations admitting a Lax Representation are generally Hamiltonian dynamics on infinite dimensional weakly-symplectic manifolds, so that the natural arena, for the analysis of their integrability, is represented by the phase space with its natural symplectic structure. In terms of this structure, the scattering data associated to the Lax operator have a natural interpretation as action-angle type variables 35].

A further progress, in the analysis of the integrability, was the important remark that many of previous systems are Hamiltonian dynamics with respect to two compatible symplectic structures [25, 26, 17, 33], this leading to a geometrical interpretation of the so called recursion operator [22].

This fact suggested that the integrability of non linear field theories could be naturally explained in terms of mixed tensor fields, whose relation [22] with Lax operators is still [8] unclear.

As a matter of fact, a description of integrability [26, 8, 15, 11, 12, 20, 23], which does not depend in a crucial way on dimensionality, and so works both for systems with finitely many degrees of freedom and for field theory can be given in terms of invariant mixed tensor field, having bidimensional eigenspaces and vanishing Nijenhuis torsion [30].

The analysis of the integrability realized with the help of a such tensor field leads to the formulation of an integrability criterion [8, 11, 12, 23] which, for finite-dimensional systems, is essentially equivalent to the classic 
Liouville theorem.

To be more specific, the mentioned essential equivalence means that the equivalence holds for non resonant Hamiltonian systems, i.e., for completely integrable dynamics whose Hamiltonian expressed in action-angle coordinates has a non vanishing Hessian.

One reason for a completely integrable Hamiltonian system to be resonant may be that the number of first integrals, defined on the entire phase space, is larger than one half of the phase space dimension (of course, in this case not all the integrals are in involution and one will have to deal with noncommuting sets of first integrals). This happens for the Kepler dynamics which, however, is bihamiltonian and has a recursion operator with the right properties [27].

More in general, the analysis of symmetries 19] shows that generally one is faced with a non Abelian algebra corresponding, for Hamiltonian systems, to a non Abelian algebra of first integrals.

The integrability of such systems, with finitely many degrees of freedom, has been analyzed in several papers [29]. There exist field dynamics, related to vector and matrix nonlinear Schroedinger equation [21, 18], possessing a noncommutative set of first integrals so that it would be useful to have a noncommutative integrability criterion formulated in terms of a recursion operator. In this paper such a criterion is presented. More specifically, in section 2 integrability criteria for the commutative case are recalled. Section 3 is devoted to noncommutative integrability. After a review of known results a new noncommutative integrability criterion is presented. The Kepler dynamics is discussed as an example.

\section{Commutative integrability criteria}

The best known criterion of integrability goes back to the celebrated Liouville theorem [24] and can be reported [3, 32, 2, 5] as follows:

Theorem If on a $2 n$ dimensional symplectic manifold $\mathcal{M}$ are defined a Hamiltonian dynamics and $n$ functionally independent first integrals $f_{1}, \ldots, f_{n}$ in involution

$$
\left\{f_{i}, f_{j}\right\}=0 \quad \forall i, j=1, \ldots, n,
$$

whose associated Hamiltonian fields $X_{i}$ are complete, then the level manifolds

$$
\mathcal{M}_{f(\pi)}=\left\{p \in \mathcal{M}: f_{i}(p)=\pi_{i} \quad i=1, \ldots, n\right\}
$$


are invariant with respect to the dynamics and each of their connected components is diffeomorphic either to $T^{m} \times \Re^{n-m}$ or, if compact, to a torus $T^{n}$. Moreover, for every point $p \in \mathcal{M}$ near which $m$ is constant, there exists a neighborhood $\mathcal{U}$ invariant under the composed flow of the vector fields $X_{i}$, and canonical coordinates $\left(P_{1}, \cdots, P_{n}, Q^{1}, \cdots, Q^{n}\right)$, where $Q^{1}, \cdots, Q^{m}$ are angles, such that the equation of the motion take the form:

$$
\dot{P}_{i}=0, \quad \dot{Q}^{i}=\nu^{i}(P), \quad 1 \leq i \leq n .
$$

A more general setting for the commutative integrability is the following [23, 34.

Let $\mathcal{M}$ be a smooth $2 n$-dimensional manifold. Let us suppose we can find $n$ vector fields $X_{1}, \ldots, X_{n} \in \mathcal{X}(\mathcal{M})$ and $n$ functions $f_{1}, \ldots, f_{n} \in \mathcal{F}(\mathcal{M})$ with the following properties

$$
\begin{gathered}
{\left[X_{i}, X_{j}\right]=0,} \\
L_{X_{i}} f^{j}=0 . \quad i, j \in\{1, \ldots, n\} .
\end{gathered}
$$

It can be shown that, if on an open dense submanifold of $\mathcal{M}$

$$
\begin{aligned}
& X_{1} \wedge \cdots \wedge X_{n} \neq 0, \\
& d f^{1} \wedge \cdots \wedge d f^{n} \neq 0,
\end{aligned}
$$

any dynamical system $\Delta$ on $\mathcal{M}$ which is of the form

$$
\Delta=\sum_{i=1}^{n} \nu^{i} X_{i}, \quad \nu^{i}=\nu^{i}\left(f^{1}, \ldots f^{n}\right)
$$

is completely integrable on the submanifold on which Eqs. (3) and (4) are satisfied.

If the fields $X_{i}$ are complete, by using the $n$-functions $f^{1}, \ldots, f^{n}$, a family of symplectic structures can defined with respect to which the dynamics is Hamiltonian.

In the Liouville theorem [24] only the commuting first integrals and the symplectic structure are given. Of course, the commuting vector fields are constructed from them. An alternative integrability theorem, suggested by the analysis of integrable models in field theory, can be formulated [8, 11, 12] using invariant tensor fields and it reads :

Theorem (DMSV) Let $\Delta$ be a dynamical vector field on a differential manifold $\mathcal{M}$ which admits a $(1,1)$ mixed tensor field $T$ which 
- is invariant

$$
L_{\Delta} T=0
$$

- has a vanishing Nijenhuis torsion

$$
\mathcal{N}_{T}=0
$$

- is diagonalizable with doubly degenerate eigenvalues $\lambda_{j}$ whose differentials $d \lambda_{j}$ are independent at each point

Then, the vector field $\Delta$ is separable, completely integrable and Hamiltonian.

Remark We observe that the Hamiltonian character of the dynamics $\Delta$ is not assumed a priori but it follows from the properties of the tensor field $T$, so that all dynamics, satisfying the given hypotheses, result to be Liouville integrable. Integrability of dissipative dynamics can be put in the same setting by assuming [10] different spectral hypothesis for the tensor field $T$. The last formulation has the advantage of being more appropriate to deal with dynamics with infinitely many degrees of freedom (completely integrable field theories). We also observe that the Lax Representation, the powerful integration tool for such systems, may not be useful in more than one space dimension since the inverse problem in Quantum Mechanics has been solved only for 1-dimensional systems.

\subsection{From the Liouville integrability to invariant mixed ten- sor fields.}

Let us now study the problem of constructing invariant mixed tensor fields, with the appropriate properties (also called a recursion tensor field), for a given Liouville's integrable Hamiltonian dynamics $\Delta$. If $H$ is the Hamiltonian function and $\{\cdot, \cdot\}$ denotes the Poisson bracket, we have

$$
\Delta f=\{H, f\} .
$$

Let us introduce in some neighborhood of a Liouville's torus $T^{n}$ actionangle coordinates $\left(J_{1}, \ldots, J_{n}, \varphi^{1}, \ldots, \varphi^{n}\right)$, in which we have:

$$
\begin{aligned}
\omega & =\sum_{h} d J_{h} \wedge d \varphi^{h} \\
\Delta & =\frac{\partial H}{\partial J_{h}} \frac{\partial}{\partial \varphi^{h}} .
\end{aligned}
$$

Let us distinguish two cases: 
- The Hamiltonian $H$ is a separable one

$$
H=\sum_{k} H_{k}\left(J_{k}\right)
$$

In this case a class of recursion tensor fields can be easily defined

$$
T=\sum_{h} \lambda_{h}\left(J_{h}\right)\left(d J_{h} \otimes \frac{\partial}{\partial J_{h}}+d \varphi^{h} \otimes \frac{\partial}{\partial \varphi^{h}}\right)
$$

with the $\lambda$ 's arbitrary and functionally independent. Indeed, the tensor field $T$ is invariant and has vanishing Nijenhuis torsion and doubly degenerate eigenvalues.

- The Hamiltonian has a non vanishing Hessian:

$$
\operatorname{det}\left(\frac{\partial^{2} H}{\partial J_{h} \partial J_{k}}\right) \neq 0 \text {. }
$$

In this case, in the chosen neighborhood, setting

$$
\nu^{h}(J)=\frac{\partial H}{\partial J_{h}}
$$

new coordinates $(\nu / \varphi)$ can be introduced, so that the dynamics can be described, with respect to the new symplectic structure

$$
\omega_{1}=\sum_{h} d \nu^{h} \wedge d \varphi^{h}=\sum_{h k} \frac{\partial^{2} H}{\partial J_{h} \partial J_{k}} d J_{k} \wedge d \varphi^{h},
$$

by a separable Hamiltonian function:

$$
H_{1}=\frac{1}{2} \sum_{h}\left(\nu^{h}\right)^{2}
$$

As before, a class of recursion tensor fields is then given by

$$
T=\sum_{h} \lambda_{h}\left(\nu^{h}\right)\left(d \nu^{h} \otimes \frac{\partial}{\partial \nu^{h}}+d \varphi^{h} \otimes \frac{\partial}{\partial \varphi^{h}}\right) .
$$

By means of this construction it is possible to find the second symplectic structure for a completely integrable Hamiltonian system.

It is still an open problem if this is true also in the remaining cases. In this direction one may find useful hints in [6, 13].

Next section is concerned with noncommutative integrable dynamics and, in particular, with their characterization in terms of an invariant, mixed tensor field. 


\section{Noncommutative integrability criteria.}

As it has been observed in the Introduction, if the number of independent first integrals is larger than half the dimension of the symplectic manifold, they cannot be in involution anymore and one will have to deal with noncommuting sets of first integrals. For a finite number of degrees of freedom a noncommutative generalization of Liouville theorem is the following [29, 4]:

Theorem (MF1) A Hamiltonian vector field on a symplectic manifold $(\mathcal{M}, \omega)$ having a noncommutative Lie algebra $\mathcal{A}$ of first integrals satisfying the condition

$$
\operatorname{dim} \mathcal{A}+\operatorname{rank} \mathcal{A}=\operatorname{dim} \mathcal{M},
$$

where $n$-rank $\mathcal{A}$ is the maximum of the rank of the matrix $m_{a b}=\left\{f_{a}, f_{b}\right\}$ 円, is completely integrable, i.e. the joint level surfaces of the first integrals are invariant, and in a neighborhood of each invariant surface one can define canonical coordinates $(\lambda / \chi / p / q)$, the $\chi$ 's being the coordinates on the invariant surfaces, such that Hamilton's equations take the form

$$
\dot{\lambda}_{i}=0, \dot{\chi}^{i}=\nu_{i}, \dot{p}_{\alpha}=0, \dot{q}^{\alpha}=0, \quad 1 \leq i \leq r, \quad r+1 \leq \alpha \leq n,
$$

with $r=\operatorname{rank} \mathcal{A}$. If these invariant surfaces are compact and connected one can prove, as in the commutative case, that they are tori, and the $\chi$ 's can be chosen to be angle variables. The canonical coordinates are called, in this case, "generalized action- angle variables". The Liouville theorem can be recovered [5] as stated by:

Theorem (MF2) If $\mathcal{M}$ is compact, then, under the hypotheses of the previous theorem, one can find $n=\frac{1}{2} \operatorname{dim} \mathcal{M}$ first integrals which are in involution. Even in this case, however, the noncommutative theorem, showing the full symmetry of the system, remains of interest.

A full account of the relevant geometrical structures underlying the noncommutative integrability, can be found in [14, 13, 7]. Here we just give a short review of them.

A symplectic form on $\mathcal{M}$ at a point $p$ defines a skewsymmetric bilinear non degenerate form on $T_{p} \mathcal{M}$. If $W$ is a $r$-dimensional subspace of $T_{p} \mathcal{M}$, the symplectic orthogonal subspace $W^{\perp} \equiv\left\{X \in T_{p} \mathcal{M}: \omega(X, Y)=0 \quad \forall Y \in W\right\}$ has dimension $2 n-r$ and in general $W \cap W^{\perp} \neq 0$.

\footnotetext{
${ }^{1}$ For semisimple Lie algebras, this definition coincides with the usual one.
} 
Two cases are of particular interest: $r \leq n$ and $r \geq n$. If $r \leq n$ and $W \subseteq W^{\perp}, W$ is said to be isotropic; if $r \geq n$ and $W \supseteq W^{\perp}, W$ is called coisotropic. If $W$ is isotropic and coisotropic $(r=n)$ then it is called $L a$ grangian. A submanifold is called isotropic, coisotropic or Lagrangian if its tangent spaces are respectively isotropic coisotropic or Lagrangian.

In the commutative case the level surfaces of the first integrals $f_{i}$ define an invariant Lagrangian foliation $\mathcal{F}_{1}$ of $\mathcal{M}$. The Hamiltonian vector fields $X_{i}$ associated to the functions $f_{i}$ are then a basis of commuting tangent vector fields for the leaves and can be used to define local coordinates $\chi^{i}$ on the leaves. These fields also commute with the Hamiltonian vector field $\Delta$ which, consequently, can be expressed as $\Delta=\nu^{i}(f) X_{i}$. In a neighborhood of a point $p \in \mathcal{M}$, the set $(\chi / f)$ define canonical coordinates and Hamilton's equations of motion take the simple following form:

$$
\dot{\chi}^{i}=\nu^{i}, \dot{f}_{i}=0 \text {. }
$$

In the noncommutative case the first integrals $f_{a}, \quad 1 \leq a \leq 2 n-r$, still define an invariant foliation, but the leaves now have dimension $r \leq n$ and the Hamiltonian vector fields $X_{a}$, associated with the first integrals $f_{a}$, are not all tangent to the leaves. However, the condition $\operatorname{dim} \mathcal{A}+\operatorname{rank} \mathcal{A}=$ $\operatorname{dim} \mathcal{M}$ ensures, for each leaf $l$, the existence of a subalgebra $\mathcal{A}_{l}$ which commutes with $\mathcal{A}$ on $l$. The Hamiltonian vector fields $\overline{X_{i}}$, associated to a basis of $\mathcal{A}_{l}$, will give themselves a basis of tangent vector fields for $l$ and will have the property $\left.\omega\left(\overline{X_{i}}, X_{a}\right)\right|_{l}=0$, so that each leaf will be 1 isotropic. To obtain a set of canonical coordinate, in a neighborhood of a point of $l$ and eventually of the whole of $l$, one needs to exploit further properties of this isotropic foliation. At each point $p$ of $l$ consider the subspace $T_{p} l \subseteq T_{p} \mathcal{M}$ and the resulting distribution of symplectically orthogonal subspaces $\left(T_{p} l\right)^{\perp}$. Since $\left.\omega\left(\overline{X_{i}}, X_{a}\right)\right|_{l}=0$, this distribution is generated, for all leaves, by the vector fields $X_{a}$, and, furthermore, since $X_{a}$ satisfy the hypotheses of the Frobenius theorem, we obtain a second coisotropic foliation $\mathcal{F}_{2}$ whose leaves are themselves foliated by those of the first foliation $\mathcal{F}_{1}$. The regularity of this foliation follows from the independence of the functions $f_{a}$. One can now prove the existence of canonical coordinates $\left(\lambda_{i}, \chi^{i}, p_{\alpha}, q^{\alpha}\right)$, such that the symplectic structure and the dynamical vector field take the following form

$$
\omega=d \lambda_{i} \wedge d \chi^{i}+d p_{\alpha} \wedge d q^{\alpha}, \Delta=\nu^{i}(\lambda) X_{i}
$$

\footnotetext{
${ }^{2}$ In particular $\left.\omega\left(X_{i}, X_{j}\right)\right|_{l}=0$.
} 
so that the equations of motion become

$$
\dot{\lambda}_{i}=0, \dot{\chi}^{i}=\nu^{i}, \dot{p}_{\alpha}=0, \dot{q}^{\alpha}=0 .
$$

The functions $\lambda_{i}$ describe locally $\mathcal{F}_{2}$, and their associated Hamiltonian vector fields $X_{i}$ define coordinates $\chi^{i}$ on $\mathcal{F}_{1}$. The fields $X_{i}$ are independent and, since $\omega\left(X_{i}, X_{a}\right)=d \lambda_{i}\left(X_{a}\right)=0$, they are tangent to the leaves of $\mathcal{F}_{1}$, and thus commute among themselves and with $\Delta$. To understand better this canonical coordinates, one can actually observe that the momentum map $J: \mathcal{M} \rightarrow \mathcal{A}^{*}$ defined by $J: x \rightarrow \xi_{x} \in \mathcal{A}^{*}$ where $\xi_{x}(f) \equiv f(x), f \in$ $\mathcal{A}$, defines a fibration of a neighborhood $\mathcal{U}$ of a leaf of $\mathcal{F}_{2}$ with fiber $l_{x}=$ $J^{-1}\left(\xi_{x}\right)$, namely a leaf of $\mathcal{F}_{1}$. The neighborhood $\mathcal{U}$ can then be represented as $l_{x} \times S \times \mathcal{O}$, where $\mathcal{O}$ is a region in the coadjoint orbit through $\xi_{x}$ of the Lie group corresponding to $\mathcal{A}$ and $S$ is a linear manifold transverse to $\mathcal{O}$. The symplectic structure $\omega$ restricted to $\mathcal{O}$ coincides with the Lie-KirillovKostant-Souriau symplectic form; $\left(p_{\alpha}, q^{\alpha}\right)$ are canonical coordinates on $\mathcal{O}$ and $\lambda_{i}$ coordinates on $S$. It has been actually proved [13] that all what is needed for the existence of such local canonical coordinates is the double foliation, namely that $\mathcal{M}$ has an isotropic foliation such that the distribution of subspaces, symplectically orthogonal to the tangent spaces to its leaves, is integrable.

\subsection{Noncommutative integrability and invariant tensor field.}

Let us give now the following new characterization of noncommutative integrability.

Theorem Let $\Delta$ be a dynamical vector field on a 2 -dimensional manifold $\mathcal{M}$ which admits a $(1,1)$ mixed tensor field $T$ which

- is invariant

$$
L_{\Delta} T=0
$$

- is diagonalizable with only simple and doubly degenerate eigenvalues whose differentials are independent at each point $p \in \mathcal{M}$.

- has the property

$$
\mathcal{N}_{T}(\alpha, X, Y)=0
$$

$\forall X: X(p) \in S(p), \forall Y \in \mathcal{D}(\mathcal{M})$ and for all 1-forms $\alpha, S(p)$ denoting the sum of eigenspaces associated to the doubly eigenvalues of $T(p)$. 
Then, the vector field $\Delta$ is separable, completely integrable and Hamiltonian. Let $\lambda_{1}, \lambda_{2}, . ., \lambda_{r}$ be the doubly degenerate eigenvalues and $\mu_{2 r+1}, \ldots, \mu_{2 n}$ be the simple ones. Under the hypotheses, the tensor field $T$ can be written in the form

$$
T=\sum_{i=1}^{r} \lambda_{i}\left(e_{i} \otimes \vartheta^{i}+e_{i+r} \otimes \vartheta^{i+r}\right)+\sum_{\alpha=2 r+1}^{2 n} \mu_{\alpha} e_{\alpha} \otimes \vartheta^{\alpha},
$$

where the $e$ 's form a basis of eigenvectors of $T$ and the $\vartheta^{\prime} s$ are the elements of the dual basis. Thus,

$$
\begin{aligned}
& T e_{i}=\lambda_{i} e_{i}, \quad T e_{i+r}=\lambda_{i} e_{i+r}, \quad T e_{\alpha}=\mu_{\alpha} e_{\alpha}, \quad i \leq r, \quad \alpha \geq 2 r+1 \\
& T \vartheta^{i}=\lambda_{i} \vartheta^{i}, \quad T \vartheta^{i+r}=\lambda_{i} \vartheta^{i+r}, \quad T \vartheta^{\alpha}=\mu_{\alpha} \vartheta^{\alpha}, \quad i \leq r, \quad \alpha \geq 2 r+1 .
\end{aligned}
$$

The Nijenhuis torsion [30 of $T$, defined by

$$
\mathcal{N}_{T}(\alpha, X, Y)=\left\langle\alpha, \mathcal{H}_{T}(X, Y)\right\rangle
$$

with

$$
\mathcal{H}_{T}(X, Y)=[T X, T Y]+T^{2}[X, Y]-T[T X, Y]-T[X, T Y],
$$

once evaluated on the basis vector fields $\left\{e_{1}, \cdots, e_{2 n}\right\}$, gives

$$
\begin{aligned}
\mathcal{H}_{T}\left(e_{i}, e_{j}\right) & =\left(T-\lambda_{i}\right)\left(T-\lambda_{j}\right)\left[e_{i}, e_{j}\right]+\left(\lambda_{i}-\lambda_{j}\right)\left[\left(L_{e_{i}} \lambda_{j}\right) e_{j}+\left(L_{e_{j}} \lambda_{i}\right) e_{i}\right] \\
\mathcal{H}_{T}\left(e_{i}, e_{\alpha}\right) & =\left(T-\lambda_{i}\right)\left(T-\mu_{\alpha}\right)\left[e_{i}, e_{j}\right]+\left(\lambda_{i}-\mu_{\alpha}\right)\left[\left(L_{e_{i}} \mu_{\alpha}\right) e_{\alpha}+\left(L_{e_{\alpha}} \lambda_{i}\right) e_{i}\right]
\end{aligned}
$$

where $i, j \leq 2 r$ and $\alpha \geq 2 r+1$, so that the conditions on the torsion imply the following relations:

$$
\begin{aligned}
& \left(T-\lambda_{i}\right)\left(T-\lambda_{j}\right)\left[e_{i}, e_{j}\right]=0, \quad\left(\lambda_{i}-\lambda_{j}\right) e_{i}\left(\lambda_{j}\right)=0, \\
& \left(T-\lambda_{i}\right)\left(T-\mu_{\alpha}\right)\left[e_{i}, e_{\alpha}\right]=0, \quad e_{i}\left(\mu_{\alpha}\right)=e_{\alpha}\left(\lambda_{i}\right)=0
\end{aligned}
$$

It follows that for any three vector fields $e_{i}, e_{j}, e_{\alpha}$

$$
\left[e_{i}, e_{j}\right]=a e_{i}+b e_{j}+c e_{i+r}+d e_{j+r}, \quad\left[e_{i}, e_{\alpha}\right]=f e_{i}+g e_{i+r}+h e_{\alpha} .
$$

Thus, the two vector fields $e_{i}$ and $e_{i+r}$, belonging to the same eigenvalue $\lambda_{i}$, satisfy the relation:

$$
\left[e_{i}, e_{i+r}\right]=c_{i} e_{i}+c_{i+r} e_{i+r},
$$

Therefore, $\forall i \in\{1, \cdots, r\}$ the vector fields $e_{i}, e_{i+r}$ are a local basis of a 2-dimensional involutive distribution and, by Frobenius' theorem, define a 
2-dimensional submanifold of $\mathcal{M}$. In other words, they can be chosen so that, on each bidimensional manifold, coordinates $\xi^{i}, \eta^{i}$ can be found such that

$$
e_{i}=\frac{\partial}{\partial \xi^{i}}, \quad e_{i+r}=\frac{\partial}{\partial \eta^{i}}
$$

In conclusion, the relations $(10)$, which directly follows from the Nijenhuis condition, ensure the "partial" holonomicity of the basis, in which the tensor field $T$ is diagonal.

On the other hand, since

$$
d \lambda_{i}=\vartheta^{j} e_{j}\left(\lambda_{i}\right)+\vartheta^{\alpha} e_{\alpha}\left(\lambda_{i}\right)=\vartheta^{j} e_{j}\left(\lambda_{i}\right),
$$

we have, by using Eq. (10),

$$
T d \lambda_{i}=T \vartheta^{j} e_{j}\left(\lambda_{i}\right)=\vartheta^{j} \lambda_{j} e_{j}\left(\lambda_{i}\right)=\vartheta^{j} \lambda_{i} e_{j}\left(\lambda_{i}\right)=\lambda_{i} d \lambda_{i}
$$

Moreover,

$$
d \mu^{\rho} \equiv d \mu_{\rho}=\sum_{k=1}^{2 r} \vartheta^{i} e_{i}\left(\mu_{\rho}\right)+\sum_{\alpha=1}^{2 n} \vartheta^{\alpha} e_{\alpha}\left(\mu_{\rho}\right)=\sum_{\alpha=1}^{2 n} \vartheta^{\alpha} e_{\alpha}\left(\mu_{\rho}\right),
$$

By means of the above relations, it is now possible to choose a holonomic basis in such a way as $T$ has the following expression

$$
T=\sum_{j=1}^{r} \lambda_{j}\left(e_{j} \otimes \vartheta^{j}+e_{r+j} \otimes d \lambda^{j}\right)+C_{\rho}^{\sigma} e_{\sigma} \otimes d \mu^{\rho},
$$

with

$$
C_{\rho}^{\sigma}=\sum_{\alpha=2 r+1}^{2 n} \mu_{\alpha} e_{\alpha}\left(\mu^{\sigma}\right)\left[e_{\alpha}\left(\mu^{\rho}\right)\right]^{-1} \quad \text { and } \quad \vartheta^{i}=0 .
$$

In addition, in a neighborhood of each bidimensional submanifold we can choose coordinates $(\lambda / \chi / \mu)$ such that the tensor $T$ can also be written in the formp:

$$
T=\sum_{j=1}^{r} \lambda_{j}\left(\frac{\partial}{\partial \lambda_{i}} \otimes d \lambda_{i}+\frac{\partial}{\partial \chi^{i}} \otimes d \chi^{i}\right)+C_{\rho}^{\sigma} \frac{\partial}{\partial \mu_{\rho}} \otimes d \mu_{\sigma} .
$$

\footnotetext{
${ }^{3}$ The symbols used for the coordinates have been choosen just to correspond to the geometric structures previously described.
} 
In the chosen basis, the vector field $\Delta$ can be written as

$$
\Delta=\Lambda_{i} \frac{\partial}{\partial \lambda_{i}}+\Phi^{i} \frac{\partial}{\partial \chi^{i}}+E^{\alpha} e_{\alpha}
$$

so that the condition $L_{\Delta} T=0$ implies that $\Lambda_{i}=E^{\alpha}=0$. It follows that

$$
\Delta=\Phi^{i}\left(\lambda_{i}, \chi^{i}\right) \frac{\partial}{\partial \chi^{i}}
$$

Symplectic structures can be found with respect to which the above vector field is Hamiltonian. Indeed, the closed 2-form

$$
\omega=\sum_{k=1}^{r} G_{k}\left(\lambda_{k}, \chi^{k}\right) d \lambda_{k} \wedge d \chi^{k}+\sum_{\alpha, \beta=2 r+1}^{2 n} f_{\alpha \beta}\left(\mu_{\alpha}, \mu_{\beta}\right) d \mu_{\alpha} \wedge d \mu_{\beta}
$$

will be invariant if

$$
\frac{\partial}{\partial \chi^{i}}\left(G_{i} \Phi^{i}\right)=0 .
$$

The non degeneracy condition for $\omega$ is obviously expressed by

$$
\operatorname{det}\left\|f_{\alpha \beta}\right\| \prod_{k=1}^{r} G_{k} \neq 0 \text {. }
$$

This is equivalent to require that if $\Phi^{i}\left(\lambda_{i}, \chi^{i}\right)$ vanishes at some point then it also vanishes on the whole integral curve of $\frac{\partial}{\partial \chi^{i}}$ through that point.

If the vector field $\Delta$ has no singular pointst, a particularly simple class of symplectic structures with respect to which it is Hamiltonian is given by

$$
\omega=\sum_{k=1}^{r} \frac{g_{k}\left(\lambda_{k}\right)}{\Phi^{k}\left(\lambda_{k}, \chi^{k}\right)} d \lambda_{k} \wedge d \chi_{k}+\sum_{\alpha, \beta=2 r+1}^{2 n} f_{\alpha \beta}\left(\mu_{\alpha}, \mu_{\beta}\right) d \mu_{\alpha} \wedge d \mu_{\beta},
$$

\footnotetext{
${ }^{4}$ If $\Phi_{k}$ is identically zero for some index $k$, we can define $\omega=\sum_{i} g_{i}\left(\lambda_{i}\right) d \lambda_{i} \wedge d \chi_{i}+\sum_{j} \frac{g_{j}\left(\lambda_{j}\right)}{\Phi^{j}\left(\lambda_{j}, \chi_{j}\right)} d \lambda_{j} \wedge d \chi_{j}+\sum_{\alpha, \beta=2 r+1}^{2 n} f_{\alpha \beta}\left(\mu_{\alpha}, \mu_{\beta}\right) d \mu_{\alpha} \wedge d \mu_{\beta}$

where the sum on the index $i$ runs over those eigenspaces for which $\Phi^{j}=0$.

When $\Delta$ has zeroes but does not vanish identically, we have to exclude this closed subset from our considerations. These sets will be invariant under the flow so that our analysis can be carried over in the same fashion as we have done on the complement.
} 
where $g_{k}$ and $f_{\alpha \beta}$ are arbitrary functions such that

$$
\operatorname{det}\left\|f_{\alpha \beta}\right\| \prod_{k=1}^{r} \frac{g_{k}}{\Phi^{k}} \neq 0 .
$$

If the submanifold $\mu=$ const is compact and connected, we can introduce, as usual, action-angle coordinates $(J, \varphi)$ so that the vector field $\Delta$ and the symplectic structure $\omega$, in the coordinates $(J, \varphi, \mu)$ take the following form:

$$
\begin{gathered}
\Delta=\Delta^{i}\left(J_{i}\right) \frac{\partial}{\partial \varphi^{i}} \\
\omega=\sum_{k=1}^{r} f_{k}\left(J_{k}\right) d J_{k} \wedge d \varphi^{k}+\sum_{\alpha, \beta=2 r+1}^{2 n} f_{\alpha \beta}\left(\mu_{\alpha}, \mu_{\beta}\right) d \mu_{\alpha} \wedge d \mu_{\beta} .
\end{gathered}
$$

In this case, the family of symplectic structures with respect to which $\Delta$ is Hamiltonian is exhaustively described in [6, 13]. The tensor field $T$ can be used to generate compatible invariant symplectic structures according to

$$
\omega_{T}(X, Y)=\omega_{1}(T X, Y)+\omega_{1}(X, T Y)+\omega_{2}(X, Y)
$$

with

$$
\omega_{1}=\sum_{k=1}^{r} f_{k}\left(J_{k}\right) d J_{k} \wedge d \varphi^{k} ; \quad \omega_{2}=\frac{1}{2} \sum_{\alpha, \beta=2 r+1}^{2 n} f_{\alpha \beta}\left(\mu_{\alpha}, \mu_{\beta}\right) d \mu_{\alpha} \wedge d \mu_{\beta} .
$$

\subsubsection{From noncommutative integrability to invariant tensor fields.}

Let us suppose to have a non commutative integrable system according to the theorem MF1. By the integrability analysis, we have the symplectic structure $\omega=d \lambda_{i} \wedge d \chi^{i}+d p_{\alpha} \wedge d q^{\alpha}$ and the equations of the motion

$$
\dot{\lambda}_{i}=0, \dot{\chi}^{i}=\nu_{i}, \dot{p}_{\alpha}=0, \dot{q}^{\alpha}=0, \quad 1 \leq i \leq r, \quad r+1 \leq \alpha \leq n,
$$

or, calling $\mu$ the collection of the $p$ 's and $q$ 's, more simply

$$
\dot{\lambda}_{i}=0, \quad \dot{\chi}_{i}=\nu_{i} \quad \dot{\mu}_{\alpha}=0 .
$$

It is easily verified that the following tensor field

$$
T=\sum_{j=1}^{r} \lambda_{j}\left(\frac{\partial}{\partial \lambda_{i}} \otimes d \lambda_{i}+\frac{\partial}{\partial \chi^{i}} \otimes d \chi^{i}\right)+C_{\rho}^{\sigma}(\mu) \frac{\partial}{\partial \mu_{\rho}} \otimes d \mu_{\sigma} .
$$


is invariant and, for all diagonalizable matrix $C_{\rho}^{\sigma}(\mu)=\delta_{\rho}^{\sigma} \mu_{\sigma}$, has a vanishing torsion, provided that the Hamiltonian function is separable in the form:

$$
H=K_{1}(\lambda)+K_{2}(\mu),
$$

with

$$
K_{1}(\lambda)=\sum_{i=1}^{r} H_{i}\left(\lambda_{i}\right)
$$

If $K_{1}$ is not separable but

$$
\operatorname{det}\left(\frac{\partial^{2} K_{1}}{\partial \lambda_{j} \partial \lambda_{i}}\right) \neq 0
$$

the construction of the invariant tensor field follows strictly the lines of section 2.1.

This shows that also in the noncommutative case an invariant torsionless tensor field can be always found. Of course, such a tensor field always generates, by repeated application, Abelian algebras of symmetries. Regardless of the vanishing of the torsion on the whole space, the noncommutative features are linked to the non degenerate eigenvalues and, then, are still described by the term $C_{\rho}^{\sigma}(\mu) \frac{\partial}{\partial \mu_{\rho}} \otimes d \mu_{\sigma}$.

Example The Kepler dynamics

\section{- A Recursion operator in the commutative case.}

The vector field for the Kepler problem, in spherical-polar coordinates, for $\Re^{3}-\{0\}$, is globally Hamiltonian with respect to the symplectic form:

$$
\omega=\sum_{i} d p_{i} \wedge d q^{i} \quad i=r, \vartheta, \varphi
$$

with Hamiltonian $H$ given by:

$$
H=\frac{1}{2 m}\left(p_{r}^{2}+\frac{p_{\vartheta}^{2}}{r^{2}}+\frac{p_{\varphi}^{2}}{r^{2} \sin ^{2} \vartheta}\right)+V(r), \quad V(r)=-\frac{k}{r}
$$

In action-angle coordinates $(J, \varphi)$, the Kepler Hamiltonian $H$, the symplectic form $\omega$ and the vector field $\Delta$ become:

$$
\begin{array}{cc}
H & =-\frac{m k^{2}}{\left(J_{r}+J_{\vartheta}+J_{\varphi}\right)^{2}} \\
\omega & =\sum_{h} d J_{h} \wedge d \varphi^{h} \\
\Delta & =\frac{2 m k^{2}}{\left(J_{r}+J_{\vartheta}+J_{\varphi}\right)^{3}}\left(\frac{\partial}{\partial \varphi^{1}}+\frac{\partial}{\partial \varphi^{2}}+\frac{\partial}{\partial \varphi^{3}}\right)
\end{array}
$$


It has been shown [27] that the vector field $\Delta$ is globally Hamiltonian also with respect to the symplectic form $\omega_{1}$ :

$$
\omega_{1}=\sum_{h k} S_{k}^{h} d J_{h} \wedge d \varphi^{k}
$$

where the matrix $S$ is defined by:

$$
S=\frac{1}{2}\left\|\begin{array}{ccc}
J_{1} & J_{2} & J_{3} \\
J_{2}-J_{3} & J_{1}+J_{3} & J_{3} \\
J_{3}-J_{2} & J_{2} & J_{1}+J_{2}
\end{array}\right\| .
$$

We have:

$$
\Delta=\left\{H_{1}, \quad\right\}_{1},
$$

with Hamiltonian $H_{1}$ given by:

$$
H_{1}=-\frac{2 m k^{2}}{J_{r}+J_{\vartheta}+J_{\varphi}}
$$

and the new Poisson brackets

$$
\{f, g\}_{1}=\sum_{h k}\left(S^{-1}\right)_{h}{ }^{k}\left(\frac{\partial f}{\partial J_{h}} \frac{\partial g}{\partial \varphi^{k}}-\frac{\partial f}{\partial \varphi^{k}} \frac{\partial g}{\partial J_{h}}\right)
$$

In the original coordinates $(p, q)$ the symplectic form $\omega_{1}$ is simply written as:

$$
\omega_{1}=\sum_{i} d K_{i} \wedge d \alpha^{i}
$$

where the functions $K_{i}(p, q)$ and $\alpha^{i}(p, q)$, defined by:

$$
\left\{\begin{array}{l}
K_{1}=\frac{1}{4}\left[J_{1}^{2}+\left(J_{2}-J_{3}\right)^{2}\right](p, q) \\
K_{1}=\frac{1}{4}\left[J_{1}^{2}+\left(J_{2}-J_{3}\right)^{2}\right](p, q) \\
K_{3}=\frac{1}{2} J_{3}\left[J_{1}+J_{2}\right](p, q) \\
\alpha^{i}=\varphi^{i}(p, q)
\end{array}\right.
$$

are considered as functions of $p, q$ by means of the map $J_{i}=J_{i}(p, q), \varphi^{i}=$ $\varphi^{i}(p, q)$. As a consequence a mixed invariant tensor field $T$, defined, for non degenerate $\omega$, by: $\omega(\widehat{T} X, Y)=\omega_{1}(X, Y)$ can be constructed.

The tensor field

$$
T=\sum_{h k}\left(S_{k}^{h} d J_{h} \otimes \frac{\partial}{\partial J_{k}}+\left(S^{+}\right)_{h}{ }^{k} d \varphi^{h} \otimes \frac{\partial}{\partial \varphi^{k}}\right)
$$


has double degenerate eigenvalues and vanishing Nijenhuis torsion, the last property being equivalent to the compatibility of the symplectic structures $\omega$ and $\omega_{1}$.

\section{- A Recursion operator in the non commutative case.}

The Kepler dynamics has five first integrals given by the components of the angular momentum and the components of the orthogonal LaplaceRunge-Lenz vector.

In action-angle coordinates $(J / \varphi)$ such first integrals are given by

$$
J_{1}, J_{2}, J_{3}, \varphi_{1}-\varphi_{2}, \varphi_{2}-\varphi_{3}
$$

By using the Delauney action-angle coordinates

$$
\begin{array}{cc}
I_{1} & =J_{1}+J_{2}+J_{3} \equiv \lambda_{1} \\
I_{2} & =J_{2}+J_{3} \equiv \mu_{3} \\
I_{3} & =J_{3} \equiv \mu_{4} \\
\alpha_{1} & =\varphi_{1} \equiv \chi_{1} \\
\alpha_{2} & =\varphi_{2}-\varphi_{1} \equiv \mu_{5} \\
\alpha_{3} & =\varphi_{3}-\varphi_{2} \equiv \mu_{6},
\end{array}
$$

we can construct the invariant torsionless tensor field

$$
T=\lambda_{1}\left(\frac{\partial}{\partial \lambda_{1}} \otimes d \lambda_{1}+\frac{\partial}{\partial \chi_{1}} \otimes d \chi_{1}\right)+\sum_{\alpha=3}^{6} \mu_{\alpha} \frac{\partial}{\partial \mu_{\alpha}} \otimes d \mu_{\alpha} .
$$

\section{Conclusion}

It has been shown that also in the non commutative case a criterion of integrability can be formulated in terms of invariant "semitorsionless" $(1,1)$ tensor field in close analogy with the commutative case. Moreover, it has also been shown that in such cases a new invariant $(1,1)$ tensor field can be constructed with a vanishing Nijenhuis torsion. By using either of them, sequences of compatible symplectic structures can be constructed. 


\section{References}

[1] M. J. Ablowitz, D. J. Kaup, A. C. Newell and H. Segur, The inverse scattering transform-Fourier analysis for nonlinear problems. Stud.Appl.Math., 53 (1974) 249

[2] R. Abraham, J. E. Marsden, Foundations of Mechanics (Benjamin/ Cummings, Reading, MA, 1978).

[3] V. I. Arnold, Mathematical Methods of Classical Mechanics (Mir, Moscow 1976).

[4] M. Adams, T. Ratiu The three-point vortex problem: Commutative and Noncommutative Integrability, Contemp. Math. 81 (1988)245-257

[5] V. I. Arnold, V.V.Kozlov, A.I. Neishtadt, Mathematical Aspects of Classical and Celestial Mechanics, Dynamical Systems III (SpringerVerlag, Berlin 1988).

[6] O.I. Bogoyavlenskij, Theory of Tensor invariants of Integrable Hamiltonian Systems. I, Comm. Math. Phys., 180 (1996)529-586; Theory of Tensor invariants of Integrable Hamiltonian Systems. II, Comm. Math. Phys., 184 (1996)301-365.

[7] O. I. Bogoyavlenskij, A concept of integrability of dynamical systems Compte. Rend. Acad. Sci. Canada vol.18 (1996)163-169; Extended Integrability and biHamiltonian systems, Comm. Math. Phys., 196, n.1 (1998)19-51.

[8] S. De Filippo, G. Marmo, M. Salerno and G. Vilasi, On the phase manifold geometry of integrable non-linear field theories, Preprint IFUSA, Salerno (1982).

[9] S. De Filippo, G. Marmo and G. Vilasi, A geometrical setting for the Lax Representation. Phys. Lett. B, 117 (1983) 418

[10] S. De Filippo, G. Marmo, M. Salerno and G. Vilasi, Phase Manifold Geometry of Burgers Hierarchy, Lett. Nuovo Cimento, 37, n.3, p.105109, (1983)

[11] S. De Filippo, G. Marmo, M. Salerno, G. Vilasi, A new Characterization of Complete Integrable Systems, Il N. Cimento 83B (1984) 97-112. 
[12] S. De Filippo, M. Salerno, G. Vilasi, A geometrical Approach to the integrability of soliton equations. Lett. Math. Phys. 9 (1985) 85.

[13] F. Fassò, T. Ratiu, Compatibility of symplectic structures adapted to noncommutatively integrable systems, J. Geom. Phys. 27 (1998)199220 .

[14] A. T. Fomenko, Symplectic Geometry. Gordon and Breach 1995.

[15] B. Florko, A.Yanovski, On Magri's theorem for complete integrability, Communication JINR, Dubna, E5-83-831, (1983)

[16] C. S. Gardner, J. M. Greene, N. D. Kruskal and R. M. Miura, A method for solving the Korteweg-de Vries equation. Pyhs. Rev. Lett., 19 (1967) 1095.

[17] I. M. Gelfand, I. Ya. Dorfman, The Schouten Bracket and Hamiltonian Operators, Funct. Anal. Appl. 14 (1980) 71-74.

[18] V. S. Gerdjikov, The generalized Zakharov-Shabat System and the Soliton Perturbations. TMF 99 n. 2, (1994)292

[19] I. S. Krasil'shchik, V.V.Lichagin, A.M.Vinogradov, Geometry of jet spaces and nonlinear partial differential equations, Gordon and Breach (1986).

[20] Y. Kosmann-Schwarzbach Geométrie des systemes bihamiltoniennes Seminaire de Mathematiques Superieurs sur les systemes dynamiques nonlineaires, Pub IRMA Lille vol.2, I (1986).

[21] P. P. Kulish, Action-angle variables for a multicomponent nonlinear Schroediger equation. J.Sov. Math. 28 n. 5, (1985)705-713.

[22] P. D. Lax, Integrals of nonlinear equations of evolutions and solitary waves. Comm. on Pure and Appl. Math., 21 (1968) 467

[23] G. Landi, G. Marmo, G. Vilasi, Recursion operators: meaning and existence for completely integrable systems, J. Math. Phys. 35(2), (1994)808-815.

[24] J. Liouville, Note sur l' intégration des équations différentielles de la Dynamique. J. Math. Pures Applied. 20, 137-138 (1855); Acta Mathematica 20, (1897)239. 
[25] F. Magri, A simple model of an integrable Hamiltonian System, J. Math. Phys., 18 (1978) 163

[26] F. Magri, A geometrical approach to the nonlinear solvable equations. Lect. Notes in Phys., 120(1980) 233.

[27] G. Marmo and G. Vilasi, When do recursion operators generate new conservation laws?, Phys. Letters B, 277 (1992)137-140.

[28] G. Marmo, E. J. Saletan, A. Simoni, B. Vitale Dynamical Systems, J.Wiley 1985.

[29] A. S. Mishenko and A. T. Fomenko, Generalized Liouville Method of Integration of Hamiltonian Systems, Funct. Anal. Appl. 12, 2 (1978)113121 , and references therein.

[30] A. Nijenhuis, $X_{n-1}$ forming sets of eigenvectors. Proc. Kon.Ned. Akad. Amsterdam 54, (1951)200-212.

[31] L. D. Faddeev and L. A. Takhtajan, Hamiltonian Methods in the Theory of Solitons, Springer-Verlag, 1987.

[32] A. M. Vinogradov and B. A. Kupershmit, The structures of Hamiltonian Mechanics, Russian Math. Surveys, 32:4 (1977)177-243.

[33] G. Vilasi, On the Hamiltonian Structures of Korteweg-de Vries and sine-Gordon Theories. Physics Letters B, 94 (1980) 195.

[34] G. Vilasi, Hamiltonian Dynamics, World Scientific 1999.

[35] V. E. Zakharov and L. D. Faddeev, Korteweg-de Vries equation, a completely integrable Hamiltonian system. Funct. Anal. Appl., 5 (1971) 18. 